\title{
Puncturing of soft gels with multi-tip needles
}

\author{
Susmita Das $\cdot$ Animangsu Ghatak
}

Received: 15 September 2010/ Accepted: 6 December 2010/Published online: 29 December 2010

(C) Springer Science+Business Media, LLC 2010

\begin{abstract}
Resistance to insertion of a sharp object into a soft solid is known to depend upon the macroscopic geometry of the tool and the consequent fracture mechanisms involved. In this article, we have examined it by carrying out displacement controlled puncturing into soft, brittle polyacrylamide gels using hypodermic needles of different diameters. These experiments show that in contrast to a rigid, flat bottom punch and a punch with sharp tip, for these needles, puncturing of the gel does not occur continuously but intermittently with the fracture progressing alternately in the wedge opening and the shear fracture modes. Correspondingly, the force versus displacement plot too shows sharp increase in load and catastrophic falls, occurring at regular intervals. We have shown that these data can be used to estimate the toughness of the material in both these fracture modes. In addition, we have examined the effect of asymmetry in the needle geometry and importantly the co-operative effect of several closely spaced needles by designing multi-tip needles consisting of several tips of different diameters and relative orientations. Experiments with these needles show that compared to a conventional single tip needle of equivalent diameter, the multi-tip needles penetrate the solid at smaller load with a smaller energy cost for fracture. Importantly, fracture occurs near simultaneously in the two fracture modes, so that the oscillations in the puncturing load is arrested. The lateral fracture of the gel and the consequent excess damage too is minimized.
\end{abstract}

S. Das · A. Ghatak $(\bowtie)$

Department of Chemical Engineering, Indian Institute

of Technology, Kanpur 208016, India

e-mail: aghatak@iitk.ac.in

\section{Introduction}

Insertion of a sharp, pointed object into a soft solid is important for variety of engineering and scientific applications: drilling and puncturing of holes for fixing and anchoring of attachment devices, suturing of thin sheets [1-3], penetration of foreign object into rubber tyres [4], remote robotic surgery [5, 6], skin puncture for blood sampling, drug delivery, catheter insertion [7], and biopsy, in forensic sciences [8-10] and even in natural world as the proboscis of organisms like mosquitoes and leeches for sucking body fluid from its hosts $[11,12]$ and as the teeth of predator animals for tearing apart the body of its victim $[13,14]$. Despite ubiquity of the puncturing phenomenon, not much is known about how fracture in these different situations is influenced by various material, geometric, and dynamic parameters associated with the tool and the solid being punctured. An important aspect, which renders fracture of a solid by a sharp puncturing tool different from its conventional breakage is that here fracture does not propagate in one plane but in three dimension which results in effects not observed with one or two dimensional fracture of materials. Consequently, the three dimensional geometry of the puncturing tool influences fracture of the solid quite significantly. For example, insertion of conventional hypodermic needle into our skin causes an acute sense of pain along with irritation and tissue damage; however, a bite from a mosquito is not normally accompanied by this unpleasant experience $[15,16]$. While the conventional hypodermic needle consists of a smoothwalled cylindrical shell with a single sharp tip, the natural puncturing device of the mosquito, called "proboscis" is known to be hierarchically patterned. In fact, it consists of two different needles both of which are used for penetrating the skin albeit at two different synchronized vibrating 
frequencies [17]. In the context of fracture of soft solids, the role of the surface structures has not yet been understood, it is not known also if these structures have any co-operative effect on fracture, nor has there been any systematic experiment to understand the dynamic effect of fracture. While, it is intuitive to think that the fracture resistance of the skin is overcome easier by the proboscis, how exactly the natural puncturing device results in a different stress field in the tissue from that of a conventional needle, is not known $[15,18]$. Nevertheless, the shape, size, and structural features of a tool are important parameters $[19,20]$, the role of which in puncturing of a soft solid needs to be investigated in systematic experiments. In this article, we have carried out puncturing of a model soft solid: poly(acrylamide) gel using conventional needles of different diameters. The poly(acrylamide) gel has the advantage that it is optically transparent and brittle with low shear modulus which allows the propagation of fracture to be visualized and monitored using a high frame rate camera $[21,22]$. In order to investigate the effect of spatial asymmetry in the stress field, we have designed multiple tip needles consisting of a single cylindrical shell fitted with several needle tips at desired orientations. We have shown that for the conventional hypodermic needle, the gel does not puncture continuously but intermittently with periodic bursts of strain energy, which we have quantified to obtain estimates of toughness of the gel in the shear fracture and the wedge opening modes. Furthermore, experiments with multi-tip needles have revealed that when the constituent needles are closely spaced, the load required to puncture the solid decreases with the expense of smaller fracture energy, suggesting a co-operative effect among the needle tips which has not been seen with conventional single tip needles. We have determined the spatial distance between these needles over which such co-operation becomes effective.

\section{Material and methods}

Semi-infinite block of polyacrylamide gel samples were prepared by polymerizing Acrylamide monomer in water, with $N N^{\prime}$-Methylene bis-Acrylamide as the cross-linker ( $0.267 \%$ by weight of the monomer), TEMED as the promotor $(0.004 \%)$, and Ammonium per sulfate $(0.04 \%$ by weight of the monomer) as the initiator. The prepolymer solution was poured into rectangular molds with dimension $20 \times 20 \times 20 \mathrm{~mm}$ which was then crosslinked over 15-20 min. In order to obtain gels of different modulus $\mu$, the monomer to water ratio was varied such that the final product contained $65-90 \%$ by weight of water. The shear modulus of the resultant gel samples were estimated as $35-5.5 \mathrm{kPa}$ [23]. The needles used for experiments were either the conventional ones with single tip or they consisted of multiple tips as shown in Fig. 1b-h. Needles of variety of outer diameter $d=0.45-1.6 \mathrm{~mm}$ and wall thickness $t=0.1-0.17 \mathrm{~mm}$ were used for generating these multi-tip needles. The tip portion of the constituent needles were closely packed at desired relative orientation and glued strongly to prevent relative motion. The diameter of the needles were at least an order of magnitude smaller than that of the gel blocks. Figure 1a shows the schematic of the experiment in which the gel sample was placed on a rigid substrate attached to a load cell while the needle was driven vertically down into it at a controlled speed.

\section{Single-tip needle}

The sequence of optical micrographs in Fig. $1 \mathrm{j}-\mathrm{o}$ depicts initiation and propagation of fracture of a block of gel when a single tip needle penetrates into it at a uniform speed; the corresponding load versus displacement plot is presented in Fig. 1i. The images in Fig. 1j, k show that the tip of the needle first indents against the surface of the gel; eventually, a threshold load is reached at which the gel surface ruptures and the needle punctures into it (micrograph k). Correspondingly, the load decreases slightly with the appearance of a peak. The rupture of the gel surface occurs via appearance of a planer mode I crack. Similar fracture mode has been observed also for initial puncture of a soft solid with a sharp tip punch and even a flat bottom cylindrical punch [19]. However, following this initiation, the crack propagates differently for the hypodermic needle than either of the sharp tip or the flat bottom punch. Whereas for the sharp tip punch, it has been observed that the crack propagates in wedge opening mode slightly ahead of the tip with continuous driving of the punch into the solid, for the flat bottom punch the crack propagates in mode II with the formation of a compressed column. Nevertheless, for both these two cases the crack propagates continuously with continuous increase in the puncturing load. However, for the hypodermic needle, the crack propagates intermittently although the needle is continued to be driven into the gel. In a typical crack pinning and propagation cycle, the fracture initially occurs in the wedge opening mode with the crack tip moving axially with the needle (micrograph 1). Close to the needle tip, a secondary crack appears which propagates in the radial direction albeit not remaining symmetric about the needle. Concomitant to it, the gel at the vicinity of the needle deforms under axial compression with corresponding increase in the load on the needle $(\mathrm{k} \rightarrow 1)$. The load eventually attains a threshold value, $F_{\max }$ at which fracture no longer occurs in the opening mode alone, as the secondary crack culminates into shear fracture; as a result, the gel block slides past the 

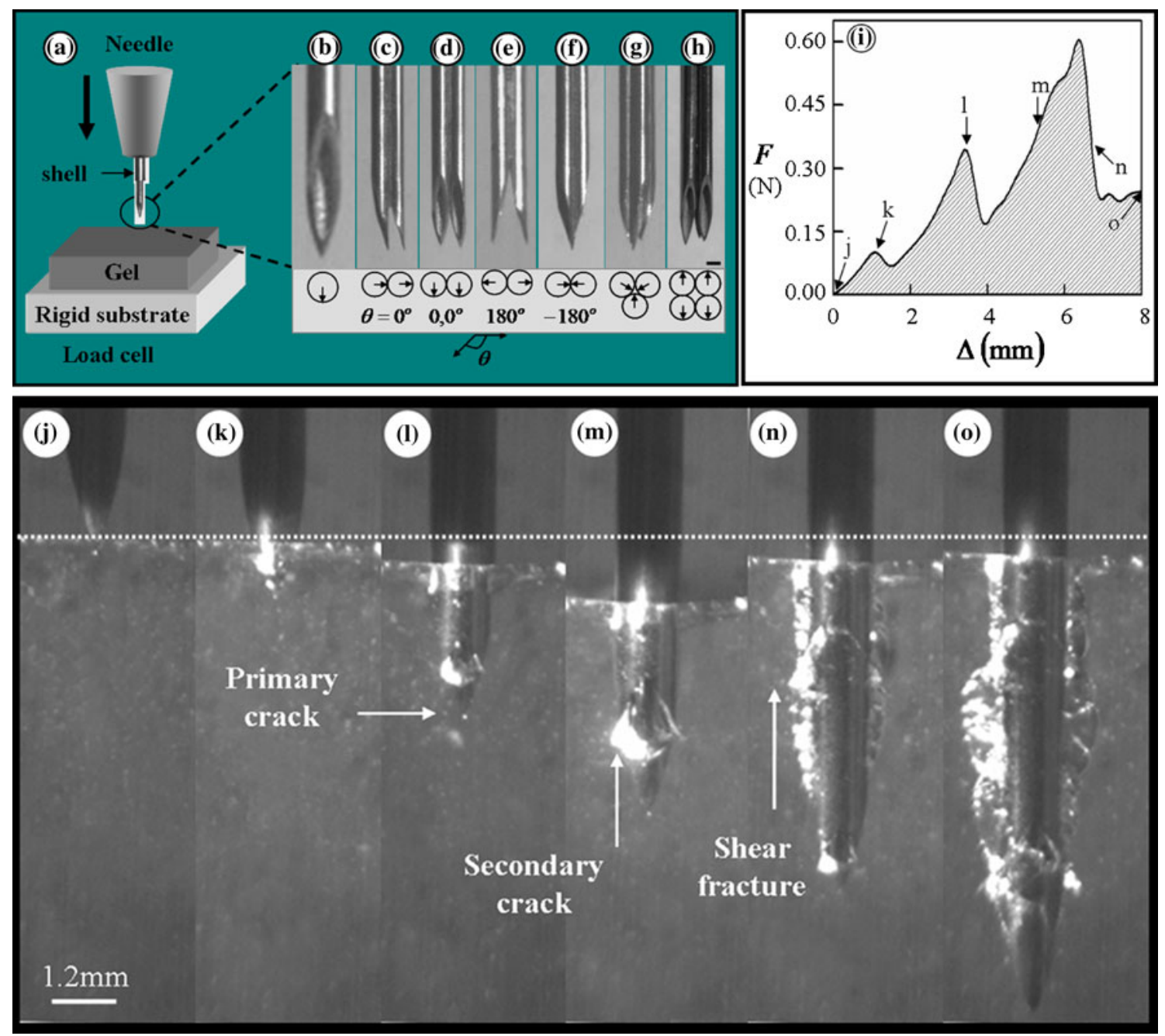

Fig. 1 (Color online) a Experiment: a gel block placed on a rigid substrate is punctured with a needle driven at a uniform speed by a motorized stage. A load cell is used to measure the dynamic load of fracture. $\mathbf{b}-\mathbf{h}$ Optical images of typical single and multi tip needles used in these experiments. b: a single tip needle; $\mathbf{c}-\mathbf{f}$ : needles with two closely packed tips oriented in different relative angles; g: needle with three closely packed tips; $\mathbf{h}$ : needle with four tips with two

cylindrical wall of the needle (Fig. 1n) catastrophically $\sim 20 \mathrm{~mm} / \mathrm{s}$, at a speed much larger than the driving speed of the needle: $0.5 \mathrm{~mm} / \mathrm{s}$. Correspondingly, the load decreases abruptly with release of burst of strain energy $(\mathrm{n} \rightarrow \mathrm{o})$. The load however does not decrease to zero but to a finite minimum value $F_{\text {min }}$. With further increase in puncturing depth $\Delta$, the crack tip moves slightly ahead in the wedge opening mode, but the needle soon catches up with it. Eventually, the secondary fracture and then the shearing fracture ensues again at $F_{\max }$ and the cycle continues. Experiments over a large range of needle speed: $0.005-1 \mathrm{~mm} / \mathrm{s}$ show that oscillations in puncturing load occur at all speeds (Fig. 2b) which suggests that these oscillations are engendered more by the fracture geometry double tips placed side by side. The circles with arrows drawn inside denote the relative location of the sharp tip of the constituent needles. i A typical plot of load versus puncturing depth into the gel shows oscillations. $\mathbf{j}-\mathbf{0}$ Video micrographs show a typical sequence of the puncture of a gel (modulus $\mu=30 \mathrm{kPa}$ ) when a single tip needle (diameter $d=1.2 \mathrm{~mm}$ ) is driven into it at $0.5 \mathrm{~mm} / \mathrm{s}$. The dashed line represents the original position of the gel surface

rather than the driving speed of the tool. To study the effect of geometry alone, we have performed all puncturing experiments at $0.5 \mathrm{~mm} / \mathrm{s}$. The optical micrograph in Fig. 3A shows the typical top view of the punctured hole on the surface of the gel block for a single tip needle of diameter $d=1.6 \mathrm{~mm}$ here the punctured hole attains a near elliptic shape which remains symmetric about the $y$ axis. The image shows that the diameter of the punctured hole is somewhat larger along the major axis $x x^{\prime}$ with two sharp corners at the ends. These features represent fracture in the wedge opening mode, i.e., in mode I. Along the $y$ axis ,however, the diameter of the hole is reduced, which implies that the needle exerts axial compression so that after it is withdrawn, the gel block relaxes back resulting in 
decrease in the diameter of the hole. We can obtain the actual area $A_{\text {actual }}$ of these non-circular holes from the images as in Fig. 3A, which yield an estimate of the effective diameter $2 r_{\mathrm{h}}=2\left(A_{\text {actual }} / \pi\right)^{1 / 2}$ of the holes. For needles of different diameters, this estimate of hole diameter was found to be $\sim 5-7 \%$ smaller than that of the outer diameter of the needle.

In the $F$ versus $\Delta$ plots, the sharp peaks and catastrophic drops occur at a regular interval $\lambda$, all of which attain a steady value only after the conical portion of the needle is completely inserted into the gel; for needles of different diameter, the length of this conical portion is found to be $\sim 4 d$. In Fig. 2a, the curves 1 and 2 for a gel of modulus $\mu=30 \mathrm{kPa}$ and needles of diameter $d=0.45-0.55 \mathrm{~mm}$ show that both wavelength and amplitude of these oscillations increase with needle diameter $d$. Curves 3 and 5 for $d=0.9$ and $1.6 \mathrm{~mm}$ puncturing into gel of $\mu=35 \mathrm{kPa}$ too exhibit the similar trend. Thus $\lambda$ increases with the diameter of the needles but decreases with the modulus of the gel as evident from the experimental data presented in Fig. 2c: $\lambda=9.8(d / t \mu)-0.39$. Notice that the slope has a unit of $\mathrm{J} / \mathrm{m}^{2}$. The intercept of the above relation predicts also that the $\lambda$ vanishes as $d$ decreases beyond a critical value $d_{\mathrm{cr}}=0.04 t \mu$; For $t=100 \mu \mathrm{m}$ and $\mu=30 \mathrm{kPa}, d_{\mathrm{cr}}$ is calculated as $120 \mu \mathrm{m}$ below which the needle would puncture the gel smoothly without any oscillations in load. The $F$ versus $\Delta$ plots yield also the maximum and minimum puncturing load data $F_{\max }$ and $F_{\min }$, which are obtained by averaging over several experiments. These data show that $F_{\max }$ increases with the diameter of the needle $(d=0.45-1.6 \mathrm{~mm})$ and the modulus of the gel $(\mu=23-35$ $\mathrm{kPa}): F_{\max }=0.027(\mu d)-0.21$ (Fig. 2d). Similarly, $F_{\min }$ data vary as $F_{\min }=0.0093(\mu d)-0.0248$

These observations can be rationalized by considering that fracture occurs in both mode-I and mode-II, although the intermittent propagation of the crack and the periodic variation of the puncturing load suggest that fracture primarily occurs in mode II during the catastrophic propagation at the threshold load $F_{\max }$ and in mode I soon after. An estimate of $F_{\max }$ can be obtained by equating the work done by the load in driving the needle by an elemental distance $\delta \Delta: F_{\max } \delta \Delta$ to the shear energy released due to fracture and the strain energy stored in the gel. Assuming that puncturing of the gel results in a cylindrical hole of radius $r_{\mathrm{h}}$, the released energy over a depth of penetration $\delta \Delta$ can be written as $\Pi_{1}=2 \pi r_{\mathrm{h}} \delta \Delta G_{\mathrm{s}}$, where, $G_{\mathrm{s}}$ is the shear fracture toughness of the gel. The stored strain energy comprises of the following two components:

(a) In the portion $r>\left(r_{\mathrm{h}}+t\right) \approx r>r_{\mathrm{h}}$ of the gel, the layer is compressed radially because of expansion of the hole diameter from $r_{\mathrm{h}}$ to $d / 2$. Notice that the co-ordinate $r$ is measured here from the axis of the needle. For the sake of simple analysis, we ignore the possible strain hardening of the gel at the threshold of the catastrophic fracture and use the neoHooken model for obtaining the strain energy density
Fig. 2 (Color online) a Force versus displacement plots for puncturing polyacrylamide gel blocks at $0.5 \mathrm{~mm} / \mathrm{s}$. Curves 1 and 2 represent puncturing a gel block of $\mu=30 \mathrm{kPa}$ with single tip needles of diameter $d=0.45$ and $0.55 \mathrm{~mm}$, respectively; similarly, curves $3-5$ represent $\mu=35 \mathrm{kPa}$ and $d=$ $0.9 \mathrm{~mm}, 23 \mathrm{kPa}$ and $1.2 \mathrm{~mm}$, and $35 \mathrm{kPa}$ and $1.6 \mathrm{~mm}$, respectively. Two dashed lines indicate the maximum and minimum puncturing loads, $F_{\max }, F_{\min }$ for curve 3. b Curves 1-4 represent data for puncturing a gel of $\mu=30 \mathrm{kPa}$ by a needle of $d=1.2 \mathrm{~mm}$ at $0.005,0.05,0.5$, and $1.0 \mathrm{~mm} / \mathrm{s}$, respectively. c The inter-peak spacing $\lambda$ varies with the scaled diameter $d / t \mu$ as

$\lambda=9.8(d / t \mu)-0.39$. d The maximum and the minimum puncturing force $F_{\text {max }}$ and $F_{\text {min }}$ vary linearly with $\mu d$
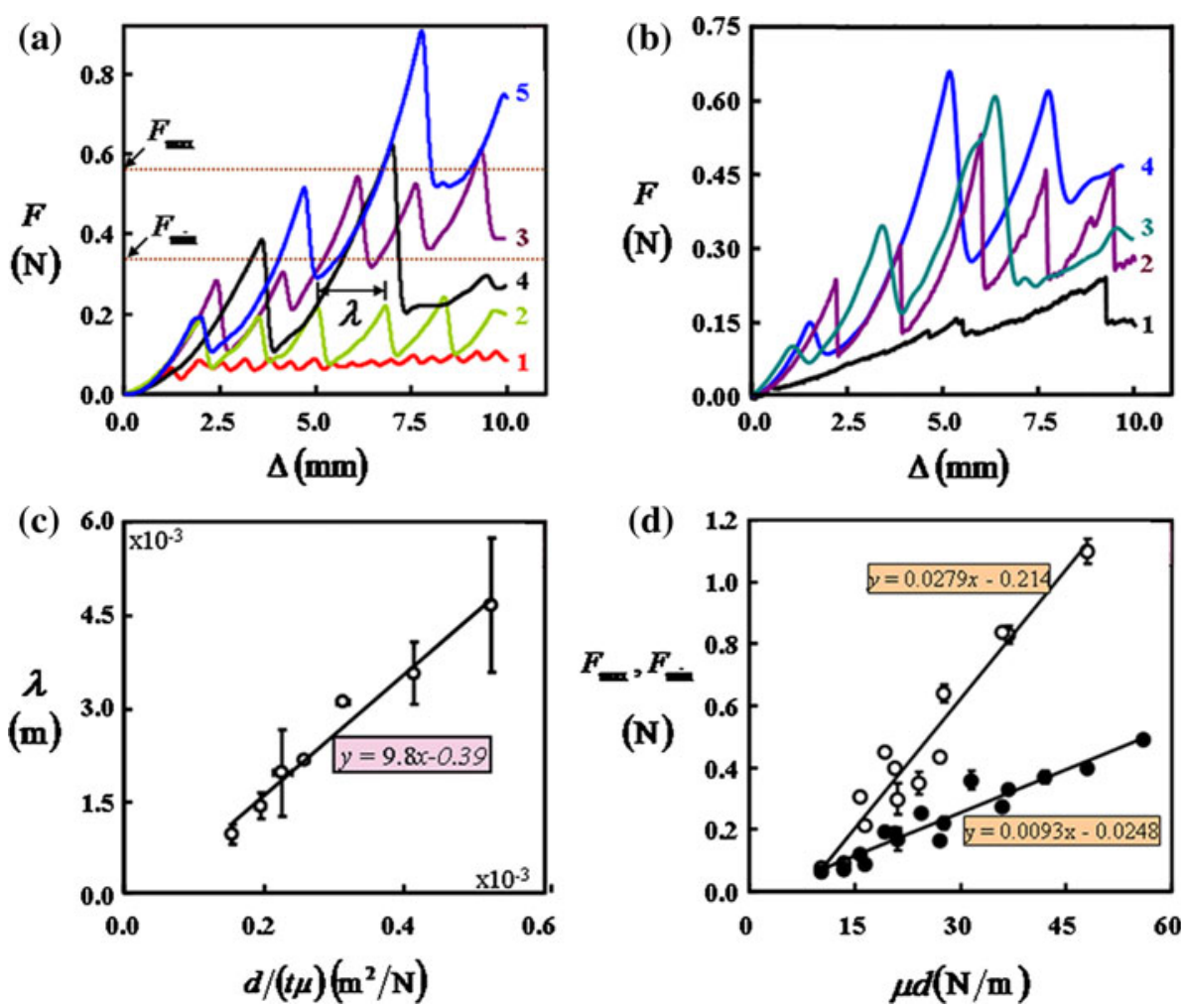


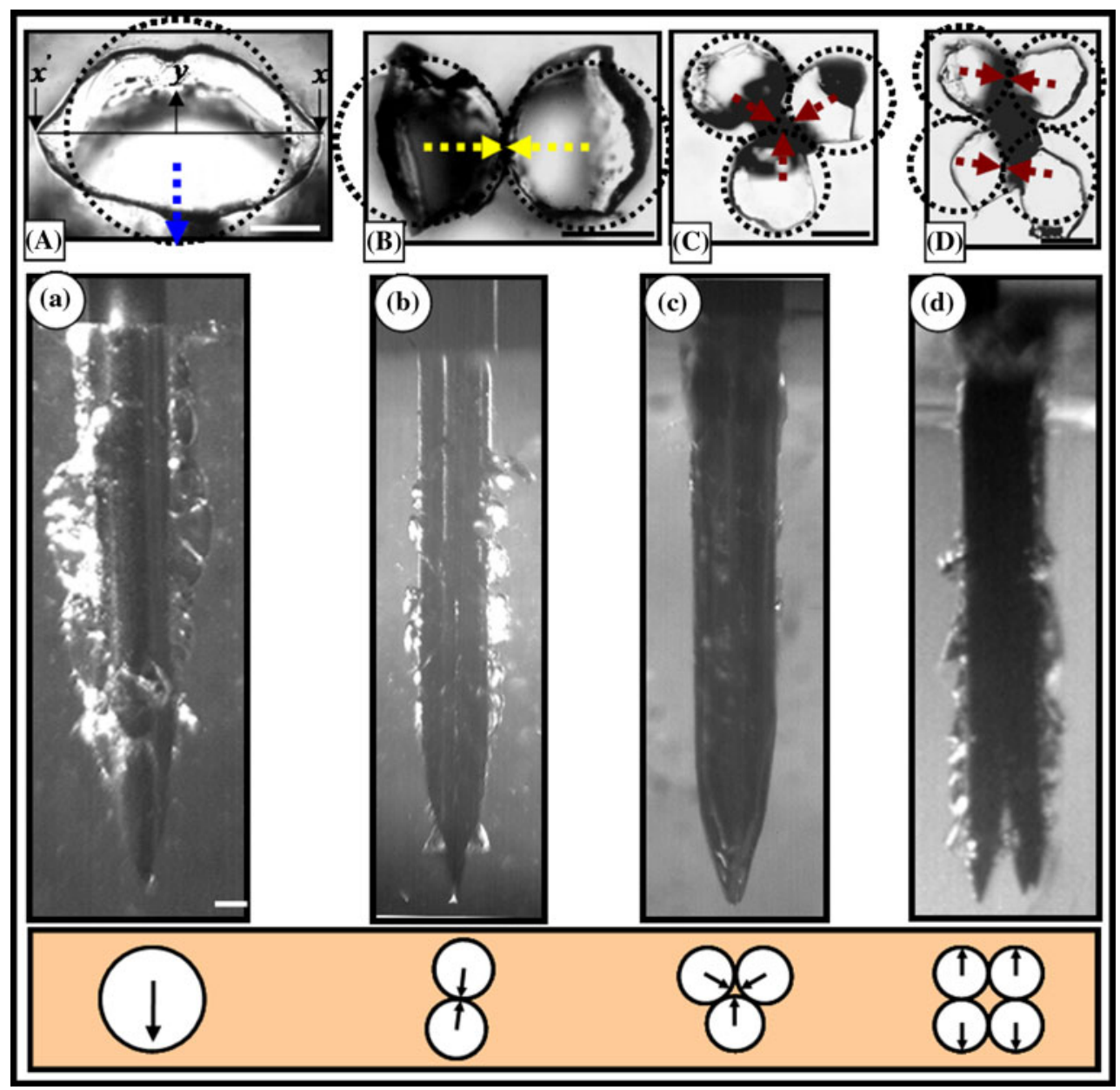

Fig. 3 (Color online) A-D Optical micrographs of punctured hole at the surface of a gel block of modulus $30 \mathrm{kPa}$. Micrograph A represents the hole with a single tip needle of diameter $1.6 \mathrm{~mm}$. B-D Micrographs are obtained for needles with 2-4 tips, respectively. These needles are constructed using individual tips of $d=0.9 \mathrm{~mm}$. The dashed circle shows the approximate position of the needle before puncture and the arrow indicates the orientation of the sharp

function, defined as $W=(\mu / 2)\left(\lambda_{r}^{2}+\lambda_{\theta}^{2}+\lambda_{z}^{2}\right)$. Here $\lambda_{r}, \lambda_{\theta}$ and $\lambda_{z}$ are the extension ratios in three orthogonal directions $r, \theta$, and $z$ such that $\lambda_{z}=1$ and due to incompressibility, $\lambda_{\theta}=1 / \lambda_{r}=r / r_{\mathrm{h}}$. If we neglect the fracture caused by the initial conical part of the needle, this component of the elastic strain energy is similar to that has been deduced for a flat bottom punch [20], therefore, for a depth $\delta \Delta$ of the gel, it is estimated as:

$\Pi_{2}=\frac{\pi \mu d^{2}}{4}\left(\frac{4 r_{\mathrm{h}}^{2}}{d^{2}}-1\right) \ln \left(\frac{2 r_{\mathrm{h}}}{d}\right) \delta \Delta$

In the portion $r_{\mathrm{h}}>r>0$, the layer is initially subjected to radial extension because of expansion of the hole from diameter $2 r_{\mathrm{h}}$ to $d$ but soon after the fracture, it relaxes back to zero load. Therefore, this portion of tip of the needles. $a-d$ Optical micrographs of side view of the fracture of a gel block of $\mu=30 \mathrm{kPa}$. Image $a$ represent puncturing of a gel using a single tip needle of $d=1.2 \mathrm{~mm}$; images $b-d$ represent fracture with double, triple, and quadruple tip needles, respectively. These multi-tip needles are constructed using tips of diameter $d=0.55 \mathrm{~mm}$. The scale bar represents $0.5 \mathrm{~mm}$

the gel does not store any strain energy unlike fracture by a flat bottom punch.

(b) The second component arises because of the finite wall thickness $t$ of the needle: the portion of the gel block underneath it, is compressed so that the extension ratios are defined as $\lambda_{r}=\lambda_{\theta}=\frac{1}{\sqrt{\lambda_{z}}}=\frac{d}{2 r_{\mathrm{h}}}$. We then obtain the following expression for the stored elastic energy [20], in the light of the neoHooken model:

$\Pi_{3}=\frac{\pi \mu d^{2}}{4}\left(2+\left(\frac{2 r_{\mathrm{h}}}{d}\right)^{6}-3\left(\frac{2 r_{\mathrm{h}}}{d}\right)^{2}\right) \frac{2 t \delta \Delta}{d}$

The summation of shear fracture energy and the stored strain energy, $\Pi_{1}+\Pi_{2}+\Pi_{3}$ is balanced with the work done by the external load to yield: 


$$
\begin{aligned}
F_{\max }= & \pi \mu d\left[\frac{d}{4}\left(\frac{4 r_{\mathrm{h}}^{2}}{d^{2}}-1\right) \ln \left(\frac{2 r_{\mathrm{h}}}{d}\right)\right. \\
& \left.+\frac{t}{2}\left(2+\left(\frac{2 r_{\mathrm{h}}}{d}\right)^{6}-3\left(\frac{2 r_{\mathrm{h}}}{d}\right)^{2}\right)+\frac{2 r_{\mathrm{h}}}{d} \frac{G_{\mathrm{s}}}{\mu}\right]
\end{aligned}
$$

We can put representative numbers from experiments to examine the relative significance of different contributions. For example, using $2 r_{\mathrm{h}} / d \sim 0.95, \mu=$ $30 \mathrm{kPa}, \quad t=0.125 \mathrm{~mm}$, and $d=0.55 \mathrm{~mm}, F_{\max }$, the above expression yields: $F_{\max }=\pi \mu d\left[4.37 \times 10^{-6}+\right.$ $\left.0.95 G_{\mathrm{s}} / \mu\right]$. Since for the gels considered $G_{\mathrm{s}} / \mu \sim$ $10^{-2} \mathrm{~m}$, the above relation signifies that the contribution from stored strain energy, i.e., $\Pi_{2}+\Pi_{3}$ is negligibly small with respect to the fracture energy $\Pi_{1}$. Nevertheless, this expression captures the linear dependence of experimental data of $F_{\max }$ on the scaled diameter of the needle $\mu d$ (Fig. 2d), from the slope of which we obtain the shear fracture toughness of the gel: $G_{\mathrm{s}}=270 \mathrm{~J} / \mathrm{m}^{2}$. It is appropriate to mention that for the brittle gel considered here, the actual fracture area exceeds that of the cylindrical hole assumed for the above calculation. As a result, underestimation of the fracture area yields a fracture toughness value which is somewhat large.

We will now show that similar to the calculation of shear toughness $G_{\mathrm{s}}$ of the gel, we can estimate also the wedge opening toughness of the gel $G_{\mathrm{w}}$, because during puncturing with a single tip needle, fracture of the gel occurs alternately in these two different modes. For example, following shear fracture of the gel at load $F_{\max }$, the load decreases to $F_{\min }$ at which the tip of the needle tears into the gel forming a mode I planer crack. The video recording of the gel fracture phenomenon shows that following appearance of a secondary wedge crack (Fig. 1m) close to the tip of the needle, fracture does not progress to any appreciable extent although the gel continues to be compressed axially with continued penetration of the needle. We therefore consider that the work done by $F_{\text {min }}$ is spent in forming a crack of length $2 a$ (Fig. 11, m) which finally can accommodate a cylinder of diameter $d$; as the needle is further driven into the gel, the load $F$ exerted on the needle increases, but the work done by it, is essentially spent in deforming the gel at its vicinity. Balancing the work done by the external load $F_{\text {min }}$ in advancing the wedge crack through an infinitesimal distance $\delta \Delta$ to the energy associated with the fracture energy of the gel: $F_{\min } \delta \Delta=2 G_{\mathrm{w}} a \delta \Delta$, we obtain the following expression for $F_{\min }$ :

$F_{\min }=\left(\frac{G_{\mathrm{w}}}{\mu d} \frac{2 a}{d}\right) \mu d$

An estimate of the crack length $2 a$ is obtained from examination of the punctured holes in the gel. For example, a typical image of a hole generated by a single tip needle as in Fig. 3A shows that along its major axis $x x^{\prime}$, the length of the mode I crack exceeds the diameter of the needle by $\sim 25 \%$, implying that $2 a / d \sim 1.2$. The slope of curve $F_{\text {min }}$ versus $\mu d$ in Fig. $2 d$ then yields $G_{\mathrm{w}} \sim 230 \mathrm{~J} / \mathrm{m}^{2}$ for a gel of shear modulus $\mu=30 \mathrm{kPa}$.

\section{Multi-tip needle}

We will now show that the gel block is punctured at a smaller load, when, instead of a single tip, we use multi-tip needles (Fig. 1c-h). The fracture surface area thus generated is $p \times \Delta$, where $p$ is the outer perimeter of the cylindrical portion of the needle and $\Delta$ is the depth of penetration. In order to compare fracture by these different needles, we can define an equivalent diameter: $d_{\text {eq }}=p / \pi$. Thus, for double, triple, and quadruple tip needles, $d_{\mathrm{eq}}$ is estimated respectively as $2 d, 2.5 d$, and $3 d$ where $d$ is the diameter of the constituent single tip needles. Hence, a triple tip needle consisting of single tips of $d=0.45 \mathrm{~mm}$ attains $d_{\mathrm{eq}}=1.13 \mathrm{~mm}$. Similarly, a single tip needle of diameter $d=1.6 \mathrm{~mm}$ is nearly equivalent to a quadruple tip generated using four tips of $d=0.55 \mathrm{~mm}$, i.e., $d_{\mathrm{eq}}=1.65 \mathrm{~mm}$. The needles of similar equivalent diameters however differ in the degeneracy of symmetry [24] because of the differing shapes of the needle cross section. If we consider only the cylindrical portion leaving aside the conical section, a single tip needle is symmetric about all directions; however, for double, triple, and quadruple tip needles, the cylindrical shell is symmetric about two, three, and four axes, respectively which implies that a double tip needle is maximally asymmetric with the level of asymmetry decreasing with increase in the number of constituent tips. The effect of asymmetry of the needle geometry is evident in top and side views of the punctured gel and the corresponding $F$ versus $\Delta$ plots.

Sequence of video-micrographs in Fig. 4a-f shows the progress of puncture of a gel block of modulus $\mu=30 \mathrm{kPa}$ as a triple tip needle of $d_{\mathrm{eq}}=1.38 \mathrm{~mm}(n=3, d=$ $0.55 \mathrm{~mm})$ is driven into it. In contrast to the single tip needles, the co-operative action of several needles result in near simultaneous fracture by different modes (opening and shear). As a result, although, both axial and lateral fracture occur in the gel, micrographs (Fig. 3a-d) show that the later remains confined to the close proximity to the needle for the multi-tip design. Micrographs (Fig. 3a) obtained for a single tip needle with $d=1.2 \mathrm{~mm}$ show an inverse conical zone which spans from the tip of the needle to the surface of the gel. While this zone does not remain perfectly symmetric about the needle, it shows that the actual fracture volume far exceeds that of the needle. 


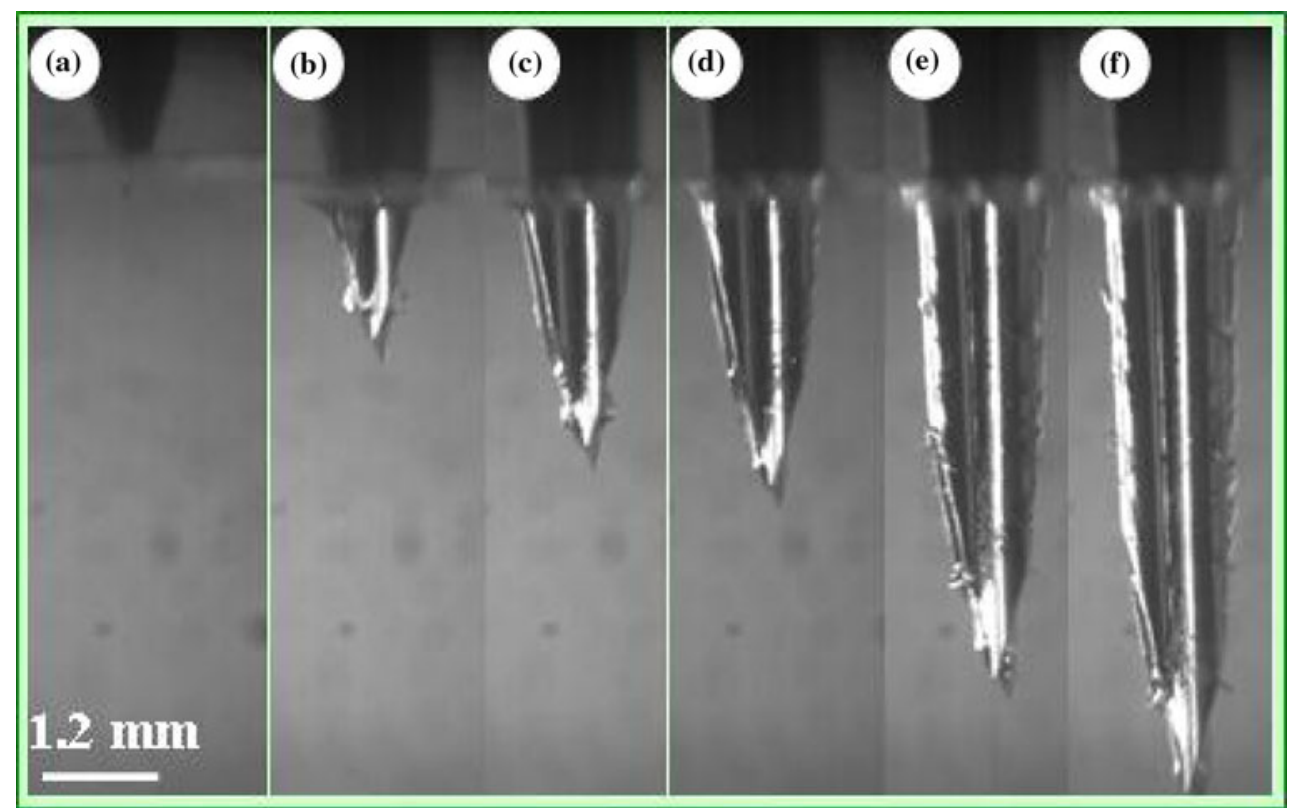

Fig. 4 (Color online) a-f Video-micrographs depict a typical sequence of fracture of an acrylamide gel of $\mu=30 \mathrm{kPa}$, when a triple tip needle of $d_{\mathrm{eq}}=1.38 \mathrm{~mm}(n=3, d=0.55 \mathrm{~mm})$ is driven into it at $0.5 \mathrm{~mm} / \mathrm{s}$

In contrast, micrographs in Fig. 3a-d show that lateral fracture diminishes as the number of constituent tips is increased from 1 to 3 , which remain closely packed with a single location for stress singularity. However, for $n=4$ two double tip needles are arranged parallel to each other resulting in two different locations of stress singularity; this arrangement results in increases in extent of lateral fracture. The shape and size of the punctured holes too appear different from that of the projected cross section of the corresponding multi-tip needle. The optical micrographs in Fig. 3A-D show the typical top views of the punctured holes for these different needles. These images are obtained after removing the needle from the punctured hole. Images in Fig. 3B-D $(n=2,3$, and 4 and $d=0.9 \mathrm{~mm})$ show that the outer perimeter of these holes exceeds that of the needles by $2-5 \%$. The area of these holes, however, is smaller than that of the needle cross section. For example, for $n=1$ and $d=1.6 \mathrm{~mm}$, the area of the punctured hole (Fig. 3A) is $15 \%$ smaller than that of the needle. For needles with increasing number of constituent tips $n=2,3$, and 4, (Fig. 3B-D) the hole area decreases by $\sim 20,23$, and $34 \%$, respectively from that of the needle. Thus, a multi-tip needle punctures a significantly smaller hole with smaller excess volume of fracture within the gel.

The $F$ versus $\Delta$ data for different multi-tip needles show that the amplitude and wavelength of oscillations change considerably from the conventional single tip design (Fig. 5a). For example, comparison of curve $1(n=1, d=0.45 \mathrm{~mm})$ of (a)

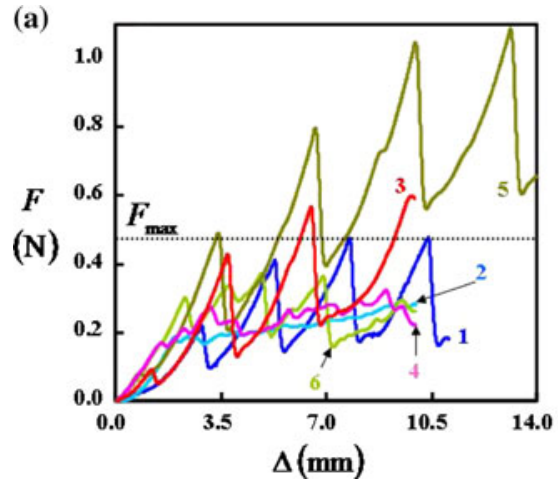

Fig. 5 (Color online) a $F$ versus $\Delta$ plots for puncturing with different multi-tip needles. Curves 1,3 , and 5 are obtained for $n=1$ and $d=0.9,1.2$, and $1.6 \mathrm{~mm}$, respectively, curve 2 : a double tip needle of $d_{\mathrm{eq}}=0.9 \mathrm{~mm}(n=2, d=0.45 \mathrm{~mm})$; curve 4 : a triple tip needle of

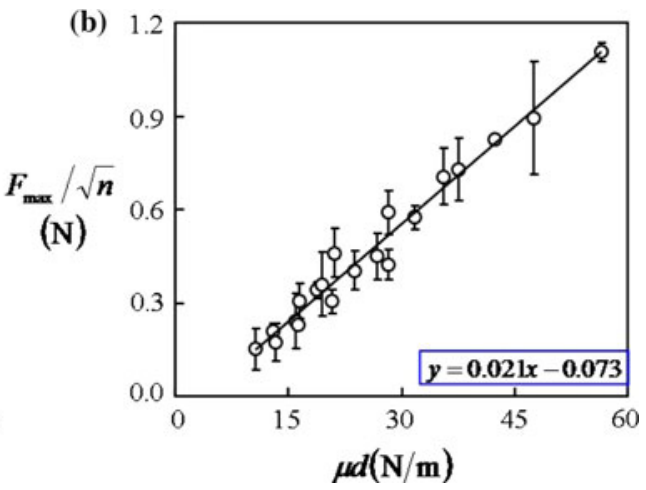

$d_{\mathrm{eq}}=1.38 \mathrm{~mm}(n=3, d=0.55 \mathrm{~mm})$; and curve 6 : a quadruple tip needle of $d_{\mathrm{eq}}=1.65 \mathrm{~mm},(n=4, d=0.55 \mathrm{~mm})$. b The scaled data of maximum puncturing load $F_{\max } / \sqrt{n}$ varies linearly with scaled equivalent diameter $\mu d$ 
Fig. 2a with curve $2(n=2, d=0.45 \mathrm{~mm})$ of Fig. $5 \mathrm{a}$, both for a gel of modulus $\mu=30 \mathrm{kPa}$, shows that with increase in number of tips $n$ from 1 to 2, amplitude of oscillations decreases, although the maximum puncture load $F_{\max }$ increases from $0.095 \pm 0.006$ to $0.25 \pm 0.04 \mathrm{~N}$; this value of $F_{\text {max }}$, however, remains considerably smaller than a single tip needle of equivalent diameter $d=0.9 \mathrm{~mm}$ as shown by curve 1 of Fig. 5a: $F_{\max }=0.44 \pm 0.01 \mathrm{~N}$. Similarly, curves 3 and 4 in Fig. 5a show that while for $n=1, d=1.2 \mathrm{~mm}$ and $\mu=30 \mathrm{kPa}, F_{\max }=0.65 \pm 0.01 \mathrm{~N}$, for $n=3$ and $d=$ $0.55 \mathrm{~mm}$, it attains a significantly smaller value, $F_{\max }=$ $0.38 \pm 0.04 \mathrm{~N}$. Importantly, this load remains nearly uniform with significantly smaller amplitude in oscillations. When we compare this data $(n=3$ and $d=0.55 \mathrm{~mm})$ with curve 2 of Fig. 2a, $(n=1, d=0.55 \mathrm{~mm})$ we see that for the latter, $F_{\max }$ is less, $0.21 \pm 0.006 \mathrm{~N}$ but the amplitude of oscillations in load is significantly large. In Fig. 5b, we show that the $F_{\max }$ data from experiments with different number of constituent needle tips fall on a single master line with the result $F_{\max } \sim \sqrt{n} d$. It signifies that for multi-tip needles, the maximum puncturing load does no longer depend upon the individual effect of the constituent needle tips which would have resulted in linear dependence on the number $n$ of constituent tips, but on the co-operative effect of the closely spaced tips. It is then expected that this effect should die down as the tips are placed increasingly further apart. In order to examine this hypothesis, we prepared needles with the gap $\delta$ between the constituent tips systematically increased by placing suitable spacers between them. Figure 6 shows the $F_{\max }$ data as a function of $\delta$ for a double tip needle. This data along with that in Fig. 5b, show that for $\delta=0, F_{\max }$ is minimum at $\left.\sqrt{2} F_{\max }\right|_{n=1}$ but increases as $\delta$ is increased, finally reaching the plateau value of $\left.2 F_{\max }\right|_{n=1}$ when $\delta$ exceeds a threshold distance of $\sim 0.3 d$. In essence, this result brings out an important point that in puncturing experiments with multi-tip needles, the co-operative action of closely spaced tips can decrease the fracture resistance of the soft solid, so that it now punctures at a smaller load.

Besides the number of tips, their relative orientation too affect the puncturing load. We have presented these different orientations for a double tip needle in Fig. 1c-f $\theta=0^{\circ},\left(0^{\circ}\right.$, $\left.0^{\circ}\right),\left(180^{\circ},-180^{\circ}\right)$. Experiments with these needles suggest that $F_{\max }$ value is minimum when the tips are packed closest, i.e., $\theta=-180^{\circ}$. In fact, oscillations in load do not diminish as much if the tips are not all closely packed as depicted by curve 6 in Fig. 5a, for $n=4, d=0.55 \mathrm{~mm}$ (here two closely packed double tip needles are glued parallel). Nevertheless, here too the peak force, $F_{\max }=0.49 \pm 0.043 \mathrm{~N}$ remains significantly smaller than that for the single tip needle of equivalent diameter $n=1, d=1.6 \mathrm{~mm}: F_{\max }=1.1 \pm$ $0.04 \mathrm{~N}$. The $F_{\max }$ data from all those experiments in which the constituent tips are closely spaced can be scaled with the
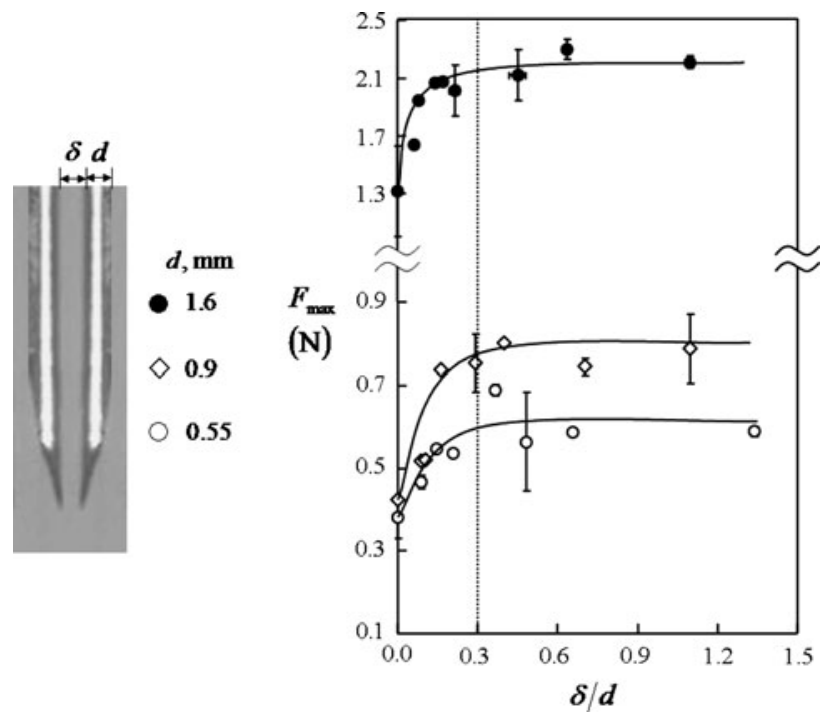

Fig. 6 The distance $\delta$ between the tips of a double tip needle is systematically varied. The plot shows the maximum puncturing load $F_{\max }$ as a function of $\delta / d$ for three different needle diameters

equivalent diameter of the needles: $F_{\max } \sqrt{n}=0.027 \mu d_{\mathrm{eq}}-$ 0.26 implying that $F_{\max }$ decreases with the number of constituent tips as $1 / \sqrt{n}$ (as shown in Fig. 7a).

We can estimate also the effective shear fracture toughness of the gel for various combinations of needle tips, following the derivation in Eq. 3 for the single tip needle. Here, we note that calculation of cross-sectional area of the punctured holes and comparison with that of the needles generate the following representative values of the ratio of effective diameter of the hole to that of the needle: $2 r_{\mathrm{h}} / d_{\mathrm{eq}}=0.9,0.88$, and 0.81 . Hence for a gel of modulus $\mu=30 \mathrm{kPa}, G_{\mathrm{s}}$ is calculated as 200,168 , and $158 \mathrm{~J} / \mathrm{m}^{2}$ for double, triple, and quadruple needles, respectively. Notice that in all these calculations, we consider the formation of a cylindrical hole due to puncturing of the gel; while this assumption is nearly consistent with the observations for $n=3$ and 4 (Fig. 3c, d, C, D), for other cases, $n=1$ and 2 (Fig. 3a, b, A, B) the actual fracture area in the gel remain somewhat larger than that of the curved surface area of the hole. It is because of this reason that the fracture toughness, an intrinsic material property, is calculated different for different combinations and arrangements of the constituent tips in the multi-tip geometry. While we do not have any measurement of the actual fracture area in these different cases, we can estimate the extent to which the fracture area decreases because of puncturing with the multi-tip needles. For example, from the $G_{\mathrm{s}}$ values for different needles, increase in fracture areas for puncturing with needles with $n=3,2$, and 1 are calculated to be 6,27 , and $71 \%$, respectively over that with $n=4$. In addition to the intrinsic toughness of the gel in the two fracture modes, we 


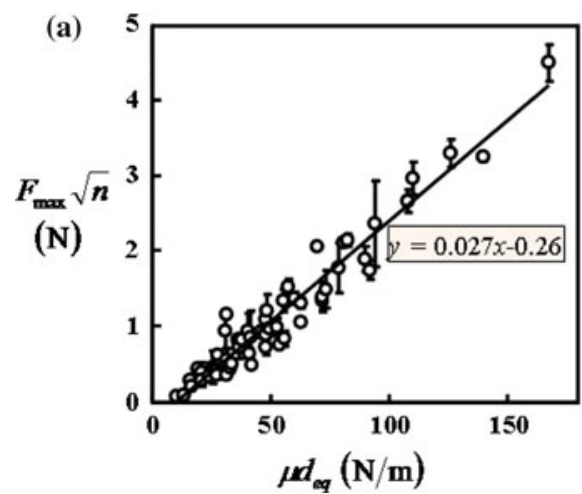

Fig. 7 a The scaled data of maximum puncturing load $F_{\max } \sqrt{n}$ varies linearly with scaled equivalent diameter $\mu d_{\text {eq. }}$. b Average fracture energy $E$ per unit depth of puncture of the gel is plotted against the equivalent diameter $d_{\mathrm{eq}}$ of the multi-tip needles. The symbols

can estimate also the net energy cost for puncturing the gel over a distance $\Delta$, by calculating the area under the $F$ versus $\Delta$ plot and averaging it over the puncture depth $\Delta: E=\int F \cdot \mathrm{d} \Delta / \Delta$. Figure $7 \mathrm{~b}$ shows the average puncturing energy $E$ for experiments with different needles and a gel of $\mu=30 \mathrm{kPa} ; E$ is largest for $n=1$, but decreases by $33-50 \%$ as the number of tips is increased to $n=2$; $E$ increases slightly for $n=3$ and 4 . These results show that the resistance to puncture decreases most when the number of tips in a multi-tip assembly is increased from $n=1$ to 2 for which the geometric asymmetry is maximized.

\section{Summary}

Decreasing the diameter of the puncturing tool has been the practice for minimizing the intensity of fracture during insertion into a soft solid as it reduces the load required to be exerted on the needle. However, decrease in diameter also leads to increase in flexibility of the needle which can result in its deviation form the intended path of insertion and serious error in placing the needle tip at a targeted location [25]. As a result, for applications, which demand the use of a long needle, the diameter simply can not be reduced. The results presented here suggest that such difficulties can be done away with, by not reducing the diameter, but by taking advantage of the three dimensional orientation of the puncturing tool. In fact, the co-operative effect of the closely spaced needles is significant as it remains effective over a large range of needle diameters and modulus of the soft gel. Such co-operation has been observed for many natural systems, e.g., school of fishes [26] has been known to reduce the muscle action by taking advantage of vortices shredded by their neighboring fishes. Similar mechanisms remain effective also for flock of birds

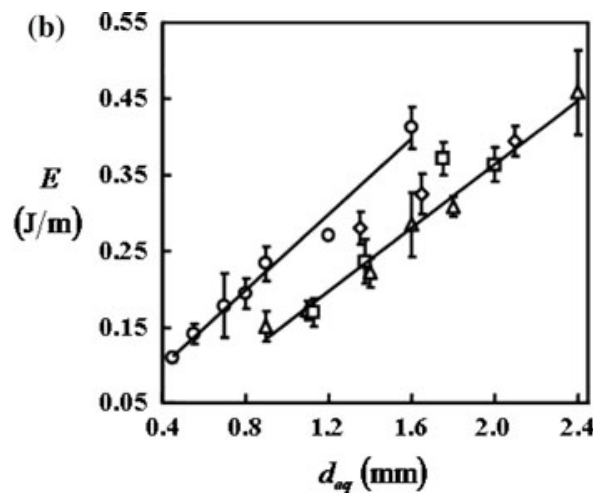

open circle, open triangle, open square, and open diamond represent data for single, double, triple, and quadruple tip needles, respectively. The error bars represent the standard deviation of data from several experiments with different needle samples

[27] and swarm of insects. In the context of the puncture experiment, how exactly the needles interact remains a subject of future interest.

Acknowledgements AG acknowledges DST, Government of India for financial support.

\section{References}

1. Fricka TB, Maruccia DD, Cartmill JA, Martin CJ, Walsh WR (2001) J Biomech 34:1335

2. Fenichel I, Oran A, Burstein G, Perry Pritsch M (2006) Int Orthop (SICOT) 3:153

3. Araki M, Tao H, Sato T et al (2009) Artif Organ 33(10):818

4. Umeno T, Asano K, Ohashi H, Yonetani M, Naitou T, Taguchi T (2001) Control Eng Prac 9(6):639

5. Brett PN, Parker TJ, Harrison AJ, Thomas TA, Carr A (1997) Proc Inst Mech Eng H 211:335

6. Bzostek A, Barnes AC, Kumar R, Anderson JH, Taylor RH (1999) In: Medical image computing and computer-assisted intervention - MICCAI99, Lecture notes in computer science (LNCS), vol 1679, p 1098

7. Stellato TA, Gauderer MW, Cohen AM (1981) Surgery 90(5):896

8. Rothschild MA, Karger B, Schneider V (2001) Forensic Sci Int 121(3): 161

9. Doberentz E, Unkrig S, Madea B (2008) Rechtsmedizin 18(6): 445

10. Wong B, Kieser JA, Ichim I, Swain M, Livingstone V, Waddell N, Taylor M (2008) Forensic Sci Med Pathol 4:212

11. Krenn HW, Plant JD, Szucsich NU (2005) Arthropod Struct Dev 34:1

12. Meyers MA, Lin AYM, Lin YS, Olevsky EA, Georgalis S (2008) JOM 60(3): 19

13. Diamond JM (1986) Nature (London) 322:773

14. Frazzetta TH (1988) Zoomorphology 108:93

15. Aoyagi S, Izumi H, Fukuda M (2008) Sens Actuators A 143

16. lzumi H, Yajima T, Aoyagi S, Tagawa N, Aral Y, Hirata M, Yorihii S (2008) IEEJ Trans Electr Electron Eng 3:425

17. Kong XQ, Wu CW (2010) Phys Rev E 82:011910

18. Dimio SP, Salcudean SE (2003) IEEE Trans Robotics Auto 19:864

19. Shergold OA, Fleck NA (2004) Proc R Soc Lond A 460:3037 
20. Shergold OA, Fleck NA (2005) J Biomech Eng 127:838

21. Livne A, Cohen G, Fineberg J (2005) Phys Rev Lett 94:224301

22. Livne A, Ben-David O, Fineberg J (2007) Phys Rev Lett 98:124301

23. Ghatak A, Das AL (2007) Phys Rev Lett 99:076101

24. Gilat G (1990) Found Phys Lett 3:189
25. Abolhassani N, Patel R (2006) In: Proceedings of the 28th IEEE EMBS annual international conference, New York

26. Liao JC, Beal DN, Lauder GV, Triantafyllou MS (2003) Science 302:1566

27. Speddingl GR, Rosén M, Hedenström A (2003) J Exp Biol 206:2313 